\title{
Effect of cryopreservation on development and ultrastructure of preimplantation embryos from the dasyurid marsupial Sminthopsis crassicaudata
}

\author{
W. G. Breed ${ }^{1}$, D. A. Taggart ${ }^{1}$, V. Bradtke ${ }^{1}$, C. M. Leigh ${ }^{1}$, \\ L. Gameau ${ }^{1}$ and J. Carroll ${ }^{2 *}$ \\ ${ }^{I}$ Department of Anatomy and Histology, University of Adelaide, Adelaide, SA 5005, Australia; \\ and ${ }^{2}$ Department of Obstetrics and Gynaecology, Queen Elizabeth Hospital, Adelaide, \\ South Australia
}

\begin{abstract}
The effect of different cryopreservation methods on the development and ultrastructure of preimplantation embryos of Sminthopsis crassicaudata, a small carnivorous marsupial and member of the family Dasyuridae, was investigated. Females were primed with $I$ iu pregnant mares' serum gonadotrophin to induce oestrus and ovulation. Mating generally ensued and, approximately 6 days after priming, embryos were collected and cultured in $5 \% \mathrm{CO}_{2}$ in air at $35^{\circ} \mathrm{C}$ for $18-22 \mathrm{~h}$ in either Dulbecco's modified Eagles medium (DMEM) with high glucose or human tubal fluid medium (HTF), both supplemented with $10 \%$ fetal calf serum. Cleavage rates were higher in DMEM than in HTF. One slow and two ultrarapid freezing methods were used. Two out of $12(17 \%)$ embryos cleaved in culture after freezing and thawing using the slow regimen, compared with six of $16(38 \%)$ non-frozen controls. In addition, two of 11 (18\%) embryos cleaved in culture following ultrarapid freezing and thawing by one of the two methods, compared to 31 of $41(76 \%)$ non-frozen controls. Most of the embryos appeared morphologically normal under the light microscope after freezing and thawing by the slow regimen, but considerable variation in the degree of ultrastructural damage to the cellular organelles was evident with the transmission electron microscope. The rather low rate of cleavage after freezing and thawing was probably due, at least in part, to ultrastructural damage of the cells.
\end{abstract}

\section{Introduction}

The ability to preserve mouse embryos at low temperatures was first demonstrated more than two decades ago (Whittingham et al., 1972; Wilmut, 1972). Since that time cryopreservation has been used for the storage of embryos from laboratory mice, domestic animals, a few rare and endangered species, and humans. Altogether, the embryos of about 15 different eutherian species have now been successfully cryopreserved (Glenister et al, 1990). However, there have been no reported attempts to extend this technique to the preservation of embryos from any marsupial species, even though this infraclass represents a major part of the Australasian and South American mammalian fauna.

The marsupial preimplantation embryo differs markedly from that of most eutherians. In the small carnivorous dasyurid marsupials, recently ovulated oocytes are $200-250 \mu \mathrm{m}$ in diameter, contain a large yolk mass that takes up much of the cytoplasmic volume and, like all marsupial oocytes so far

*Present address: MRC Unit of Experimental Embryology and Teratology, St George's Hospital Medical School, Cranmer Terrace, London, SWI7 ORE, UK.

Received 10 May 1993. examined, are not surrounded by any cumulus or corona cells (Hill, 1910; Selwood and Young, 1983; Selwood, 1987; Breed and Leigh, 1990, 1992). Fertilization occurs in the ampulla of the oviduct and within less than $20 \mathrm{~h}$ the zygotes have entered the uterus. During their passage along the oviduct, a mucoid coat is laid down (whereas in uterine eggs a shell membrane is evident outside this) and at about the time of the first cleavage division the yolk mass is extruded into the perivitelline space (Breed and Leigh, 1990, 1992). In the brown marsupial mouse Antechinus stuartii, the first and second cleavage divisions occur parallel to the polar axis of the embryo (Selwood and Young, 1983; Selwood, 1989b), and the blastomeres that are formed are initially connected by a midbody or cytoplasmic bridge. However, at the four-cell stage, the blastomeres separate, become more elongated and adhere to the inner surface of the zona pellucida (Selwood, 1989a, b; Selwood and Smith, 1990).

In this study we used the fat-tailed dunnart Sminthopsis crassicaudata, a small dasyurid marsupial, to examine the effects of cryopreservation on the ability of embryos to undergo cleavage in culture and the resultant effect on the ultrastructure of the embryonic cells. 


\section{Materials and Methods}

\section{Source of embryos}

Sminthopsis crassicaudata were obtained from the breeding colony housed in the Department of Genetics, University of Adelaide (see Bennett et al., 1990 for conditions of maintenance). Groups of separated adult females were injected with $I$ iu pregnant mares' serum gonadotrophin (PMSG) i.p., which often induces oestrus and ovulation a few days later (Rodger et al., 1992). The females were housed with sexually mature males $48 \mathrm{~h}$ after PMSG administration, and mating was determined by the occurrence of spermatozoa in the urine of the females. Animals were killed by either cervical dislocation or an overdose of sodium pentobarbitone (Nembutal, Bomac Laboratories, Sydney) 2-4 days after mating had occurred. The reproductive tracts were collected in a watchglass containing warm (i) phosphate-buffered saline (PBS; Flow Laboratories, Irvine), (ii) Hepes-buffered Dulbecco's modified Eagles medium, with high glucose and L-glutamine (DMEM; Sigma Chemical Co., St Louis MO) or (iii) Hepes-buffered human tubal fluid (HTF), (Quinn et al., 1985), all of which were supplemented with $10 \%$ fetal calf serum (Flow Laboratories). Under a dissecting microscope, the oviducts were examined and, if zygotes were seen, the tubes were torn using fine forceps and the resultant embryos expelled. The uterus was bisected and inverted in the culture dish to recover uterine embryos with the result that the embryos were released into the medium. After collection, they were transferred to a fresh dish of prewarmed medium.

\section{Cryopreservation}

Usually the technique used for embryo freezing was that of Carroll et al. (1989), but in a few experiments the methods of Kasai et al. (1990) and Shaw et al. (1991) were used.

When the method of Carroll et al. (1989) (Method 1) was used, embryos were transferred to a watchglass containing $1.5 \mathrm{~mol}$ dimethyl sulfoxide $\mathrm{l}^{-1}$ (DMSO; BDH Chemicals, Poole) on ice. After 2-5 min, the embryos and about $0.2 \mathrm{ml}$ of the freezing medium were drawn into a $0.25 \mathrm{ml}$ plastic straw (IMV Victorian Artificial Breeders, Bacchus Marsh, Victoria), which was sealed with Cristaseal (Hawkesly \& Sons, Lancing) and placed in a programmed cell freezer (Freeze Control CL 863 system with Cryogenesis software, Cryologic Pty Ltd, Mt Waverly, Victoria) at $0^{\circ} \mathrm{C}$. After equilibration for $12 \mathrm{~min}$ in DMSO, the straws were cooled at $2^{\circ} \mathrm{C} \min ^{-1}$ to $-7^{\circ} \mathrm{C}$. Ice formation was induced 2 min later by touching the top of the straw with forceps cooled in liquid nitrogen. After a further $5 \mathrm{~min}$, cooling was continued at $0.3^{\circ} \mathrm{C} \mathrm{min}^{-1}$ to $-40^{\circ} \mathrm{C}$. The straws were then transferred to liquid nitrogen and stored for up to $24 \mathrm{~h}$.

For the method of Shaw et al. (1991) (Method 2) the cryoprotectant solution contained $0.25 \mathrm{~mol}$ sucrose $1^{-1}$ and $4.5 \mathrm{~mol} \mathrm{DMSO} \mathrm{I}^{-1}$. Embryos were transferred with a minimal amount of M2 medium (Quinn et al., 1982) into the cryoprotectant solution within the insemination straw at $0^{\circ} \mathrm{C}$, using a fine glass pipette. Straws were placed on ice at $0^{\circ} \mathrm{C}$ before the transfer of embryos. After insertion of the embryos, straws were sealed as described above and then returned to the ice bed for equilibration for a further $10 \mathrm{~min}$. They were then removed, wiped dry and immediately plunged into liquid nitrogen.

For the method of Kasai et al. (1990) (Method 3), embryos were transferred into a cryoprotectant solution containing $40 \%$ ethylene glycol, $30 \%$ Ficoll and $0.5 \mathrm{~mol}$ sucrose $\mathrm{I}^{-1}$ in a modified PBS (PBS-S), at room temperature (Whittingham, 1971). After 2-5 min equilibration they were transferred into the straws, which were sealed and immediately plunged into liquid nitrogen.

\section{Thawing and dilution of cryoprotectant}

The thawing regimen varied depending upon the freezing protocol that was followed.

Embryos frozen using Method I were thawed rapidly by transferring the straw from liquid nitrogen to a water bath at $30^{\circ} \mathrm{C}$. The contents of the straw were emptied into a watchglass and DMSO was diluted by adding $0.8 \mathrm{ml}$ of Hepes-HTF; a further $1.0 \mathrm{ml}$ of culture medium was added 10 min later. Ten minutes after the last dilution, the embryos were washed in Hepes-HTF and warmed to $35^{\circ} \mathrm{C}$.

Embryos frozen following Method 2 were thawed rapidly by transferring straws from liquid nitrogen to a water bath at $35^{\circ} \mathrm{C}$ (about $5 \mathrm{~s}$ ) until the cryoprotectant solution had melted. The thawed solution containing the embryos was then expelled into medium M2 containing $0.25 \mathrm{~mol}$ sucrose $\mathrm{I}^{-1}$ and left for $10 \mathrm{~min}$ at room temperature. After a further $10 \mathrm{~min}$ in medium $M 2$, the embryos were transferred to culture medium.

Embryos frozen using Method 3 were thawed rapidly by plunging straws into a water bath at $20^{\circ} \mathrm{C}$ for $5 \mathrm{~s}$. The contents were then expelled into a $0.5 \mathrm{~mol}$ sucrose $\mathrm{l}^{-1}$ solution in PBS-S at $20^{\circ} \mathrm{C}$ for $5 \mathrm{~min}$. Embryos were then washed in fresh PBS-S and transferred to culture medium.

\section{Embryo culture}

Embryos that had been frozen-thawed using the method of Carroll et al. (1989) (Method 1) were cultured in HTF containing $10 \%$ fetal calf serum, 100 iu penicillin $\mathrm{ml}^{-1}$ and $50 \mu \mathrm{g}$ streptomycin $\mathrm{ml}^{-1}$ (Quinn $e t$ al., 1985). However, during the course of this study, a method for culturing Sminthopsis embryos was published by Selwood (1989a, b) and in subsequent experiments, this method was used and embryos were cultured in DMEM with high glucose, and L-glutamine (Sigma). Fetal calf serum (10\%), penicillin (100 iu $\mathrm{ml}^{-1}$ ), (CSL, Melbourne, Victoria) and streptomycin sulfate $\left(50 \mu \mathrm{g} \mathrm{ml}^{-1}\right)$ (Glaxo Australia, Melbourne) were also added.

All embryos were cultured for $18-22 \mathrm{~h}$ in $2 \mathrm{ml}$ of medium under mineral oil (Sigma Chemical Co.) gassed with $5 \% \mathrm{CO}_{2}$ in air and maintained at $35^{\circ} \mathrm{C}$.

\section{Morphological analysis}

Two one-cell and five two-cell embryos were exposed to the cryoprotectant solution used in Method I (Carroll et al., 1989) to determine the effect of the addition and removal of DMSO on embryo morphology. 
Table 1. Numbers and developmental stages of control embryos from Sminthopsis crassicaudata before and after culture

\begin{tabular}{lccccc}
\hline Culture media & $\begin{array}{c}\text { Number of } \\
\text { expts }\end{array}$ & $\begin{array}{c}\text { Total number } \\
\text { of embryos }\end{array}$ & $\begin{array}{c}\text { Number of } \\
\text { embryo types } \\
\text { before culture }\end{array}$ & $\begin{array}{c}\text { Number of } \\
\text { embryos lost } \\
\text { in processing }\end{array}$ & $\begin{array}{c}\text { Number of } \\
\text { embryo types } \\
\text { after culture }\end{array}$ \\
\hline DMEM & 6 & 43 & $28(1), 14(2), 1(4)$ & 2 & $\begin{array}{c}\text { Number of } \\
\text { embryos that } \\
\text { cleaved } \\
\text { HTF }\end{array}$ \\
\hline
\end{tabular}

aEmbryo type indicated in parentheses: (1), one-cell; (2), two-cell; (4), four-cell embryos.

DMEM: Hepes-buffered Dulbecco's modified Eagles medium; HTF: Hepes-buffered human tubal fluid.

Morphologically normal control, frozen-thawed and cryoprotectant-treated embryos were also fixed in a mixture of $3 \%$ glutaraldehyde and 3\% paraformaldehyde, made up in $0.1 \mathrm{~mol}$ phosphate buffer $\mathrm{l}^{-1}, \mathrm{pH} 7.4$, to which had been added $2.5 \%$ polyvinylpyrrolidone (Sigma). After fixation for $0.5-2 \mathrm{~h}$, the embryos were washed twice in buffer, placed in $1 \%$ osmium tetroxide for $1 \mathrm{~h}$, dehydrated by passing through a graded series of alcohols, and embedded in epoxy resin. Plastic sections, $0.5-1 \mu \mathrm{m}$ thick, were cut and stained with toluidine blue in borate buffer. When appropriate regions were found, ultrathin sections were cut and stained with uranyl acetate and lead citrate. The sections were observed with a JEOL $100 \mathrm{~S}$ or $2000 \mathrm{FX}$ transmission electron microscope.

\section{Assessment of embryos}

The possibility of including parthenogenetically activated embryos, which occur frequently in these animals (Anderson and Breed, 1993), was eliminated by including only those with spermatozoa found trapped within the mucoid coat in the analysis. They were usually examined with phase contrast optics using an inverted microscope before and immediately after freezing-thawing, and also after $18-22 \mathrm{~h}$ in culture if this had taken place. A qualitative morphological assessment was made of the number of intact blastomeres, their shape, the condition of the zona pellucida and shell membrane. Some embryos were also fixed for light and transmission electron microscopy.

\section{Results}

\section{Effect of cryopreservation on subsequent cleavage of embryos}

A total of 61 females was primed with PMSG to collect embryos for culture. Of these, 40 females $(66 \%)$ had spermatozoa present in the urine 3-4 days after priming. Embryos were recovered from 34 animals, although not always from those in which mating had been recorded. A total of 474 embryos was recovered, ranging from I-31 embryos per female, and the mean ovulation rate was $13.0 \pm 3.7$ embryos per female. Most embryos recovered were between the one-cell and four-cell stage of development.

Controls. In nine experiments, a total of 59 embryos at the one-four-cell stage were cultured for $18-22 \mathrm{~h}$ (Table 1).
Forty-three of these (28 one-cell, 14 two-cell and one four-cell embryos) were cultured in DMEM, and 16 (11 one-cell and five two-cell embryos) in HTF medium. Of these, 31 of $41(76 \%)$ and six of $16(38 \%)$, respectively, cleaved over the $18-22 \mathrm{~h}$ of culture (Table 1). Approximately 3 of $10(30 \%)$ of the remaining embryos cultured in DMEM had degenerated as their blastomeres had shrunk or become irregular in shape and developed a granular appearance. Of the 10 of 16 (63\%) HTF-cultured control embryos that did not divide, approximately half showed some degenerative changes similar to those described above for embryos in DMEM. The remaining control embryos that did not cleave appeared morphologically normal under the light microscope.

Frozen-thawed embryos. Thirty-six one-four-cell embryos were cryopreserved using the three methods described. After thawing, 27 embryos were recovered, placed in the appropriate medium, and cultured for 18-22 h. Four of these subsequently cleaved (Table 2).

After freezing by the method of Carroll et al. (1989) (Method 1), 2 of 12 (17\%) embryos cleaved in culture. These two embryos, both one-cell zygotes at the start of the experiment, underwent two divisions over the $18-22 \mathrm{~h}$ culture to form four-cell embryos (Table 2). The majority, about 6 of $10(60 \%)$, of the remaining embryos frozen in this manner appeared morphologically normal under the light microscope at the end of the culture period but had not cleaved. The remaining four embryos had shrunken blastomeres that often had a rather granular appearance.

No embryos frozen using the ultrarapid freezing method of Shaw et al. (1991) cleaved in culture. However, their morphology under the light microscope appeared normal at the conclusion of culture and their egg vestments were intact; blastomeres were of similar size and density and showed no obvious signs of shrinkage or degeneration.

After using the ultrarapid embryo cryopreservation method of Kasai et al. (1990) 2 of 11 (18\%) of embryos divided in culture. Both were initially one-cell zygotes and both underwent a single cleavage division to form two blastomeres each (Table 2). Of the remaining embryos approximately a third (3 of 9) appeared morphologically normal at the end of culture. The remainder showed some degree of damage from slight shrinkage of the blastomeres to substantial fragmentation of the mucoid coat and shell membrane. 
Table 2. Numbers and developmental stages of embryos from Sminthopsis crassicaudata cultured before and after cryopreservation

\begin{tabular}{|c|c|c|c|c|c|c|c|}
\hline $\begin{array}{l}\text { Freeze-thaw } \\
\text { protocol }\end{array}$ & $\begin{array}{c}\text { Number of } \\
\text { expts }\end{array}$ & $\begin{array}{l}\text { Total number } \\
\text { of embryos }\end{array}$ & $\begin{array}{l}\text { Number of } \\
\text { embryo types } \\
\text { before culture }\end{array}$ & $\begin{array}{l}\text { Number of } \\
\text { embryos recovered } \\
\text { after freezing }\end{array}$ & $\begin{array}{l}\text { Number of } \\
\text { embryo types } \\
\text { after culture }\end{array}$ & $\begin{array}{l}\text { Number of } \\
\text { embryos that } \\
\text { cleaved }\end{array}$ & $\begin{array}{l}\text { Divisions during } \\
18-22 \mathrm{~h} \text { of } \\
\text { culture }\end{array}$ \\
\hline (1) Carroll et al., 1989 & 5 & 20 & $4(1), 14(2), 2(4)$ & $12(12 / 20)$ & $3(1), 9(2), 0(4)$ & $2 / 12(17 \%)$ & $2 \times 1$-cell- $2 \times 4$-cell \\
\hline (2) Shaw et al., 1991 & 3 & 4 & $2(1), 2(2), 0(4)$ & $4 \quad(4 / 4)$ & $2(1), 2(2), 0(4)$ & $0 / 4(0 \%)$ & 0 \\
\hline (3) Kasai et al., 1990 & 3 & 12 & $3(1), 9(2), 0(4)$ & $11(11 / 12)$ & $0(1), 11(2), O(4)$ & $2 / 11(18 \%)$ & $2 \times 1$-cell-2 $2 \times 2$-cell \\
\hline
\end{tabular}

${ }^{a} E$ Embryo type indicated in parentheses: (1), one-cell; (2), two-cell; (4), four-cell embryos.

Table 3. Morphology of embryos from Sminthopsis crassicaudata after freezing and thawing (by light microscopy)

\begin{tabular}{lccc}
\hline & \multicolumn{3}{c}{ No. of embryos } \\
\cline { 2 - 4 } Stage of development & Frozen & Recovered & Normal morphology \\
\hline \multirow{2}{*}{ One-cell } & 8 & 6 & 4 \\
Two-cell & 8 & 8 & 7 \\
Four-cell & 9 & 9 & 9 \\
\cline { 2 - 4 } Total & 25 & 23 & 20 \\
\hline
\end{tabular}

\section{Light microscope studies of effect of cryopreservation on embryo morphology}

The method of Carroll et al. (1989) was used to freeze 25 embryos at the one-cell, two-cell and four-cell stages, 23 of which were subsequently recovered from the straws after thawing; 20 of these (85\%) appeared morphologically normal when observed under phase contrast optics (Table 3). Of the three damaged embryos, two had lysed blastomeres and one a disrupted shell membrane and zona pellucida.

On exposure to $1.5 \mathrm{~mol} \mathrm{DMSO}^{-1}$, the blastomeres of the two zygotes and five two-cell embryos initially shrank but after about 10 min they began to re-expand. After dilution of the cryoprotectant and equilibration in isotonic medium, the embryos expanded to their normal volume and all blastomeres subsequently appeared morphologically normal.

\section{Electron microscope studies of effects of cryopreservation on embryo morphology}

The following ultrastructural features were found in the zygotes, two-cell and four-cell embryos before and after freezing and thawing by the method of Carroll et al. (1989).

Zygotes. In the two control zygotes, there was a large 'yolk mass' in the centre and a peripheral region of cytoplasm with numerous vacuoles and abundant microvilli extending into the perivitelline space. The extracellular coats included a $2-4 \mu \mathrm{m}$ thick zona pellucida, a thin mucoid layer, and a shell membrane.

Examination of the outer cortical region of cytoplasm with the electron microscope showed abundant small mitochondria with a dense matrix, lamellar cristae, and occasional electron- dense granules. In addition, there were large vacuoles that contained flocculent material, some evidence of compartmentalization of the cytoplasm by membranes, occasional whorls or cisternae of endoplasmic reticulum, scattered lipid droplets, and a rather granular and electron-dense cytoplasmic matrix. Close to, and sometimes fusing with, the cell membrane were some coated vesicles.

In DMSO-treated embryos $(n=2)$ the ultrastructural appearance was generally similar except that the blastomeres appeared slightly shrunken, the outer cortical cytoplasm was more variable in electron density, and some mitochondria had somewhat distorted cristae.

The morphological characteristics of the four frozen-thawed zygotes varied and sometimes cytoplasm of different electron density and vacuolation was evident, especially in the region close to the cell membrane. In addition, intracytoplasmic spaces and membranous whorls were sometimes seen, and the nuclear envelope appeared slightly wrinkled. Microvilli were sometimes less prominent than they were in controls. In some of the mitochondria, the matrix was less electron-dense and lamella cristae were not always discernible. However, the structure of the large vacuoles, cisternae and reticulum appeared normal.

Two-cell embryos. In the seven two-cell control embryos, one of the blastomeres was connected to the yolk mass, which lay as a separate structure in the perivitelline space. Microvilli on the surfaces of the opposing blastomeres interdigitated. A sperm tail could sometimes still be seen and at least one of the embryos sectioned had a binuclear blastomere. The nuclei were surrounded by a vacuole-rich region, whereas the cortical cytoplasm was less vacuolated.

The outer region of the cytoplasm had variable electron density, and microvilli protruded into the perivitelline space (Fig. Ia, b, c). There were a few large vacuoles containing flocculent material and mitochondria had a dense matrix and lamellar cristae. Coated vesicles, some of which were in the process of fusing with the cell membrane, groups of lipid droplets, vesicles, and cisternae of endoplasmic reticulum were also sometimes visible (Fig. 1b, c). The nucleus had much euchromatin and an occasional prominent nucleolus. In a few embryos, the nuclear envelope had a wrinkled appearance.

In the DMSO-treated embryos $(n=5)$ the ultrastructure of the blastomeres was similar to that of controls with variable regions of electron density of the outer cortical region. Vacuoles, some with flocculent material, were common and typical scattered vesicles could be seen particularly around the 

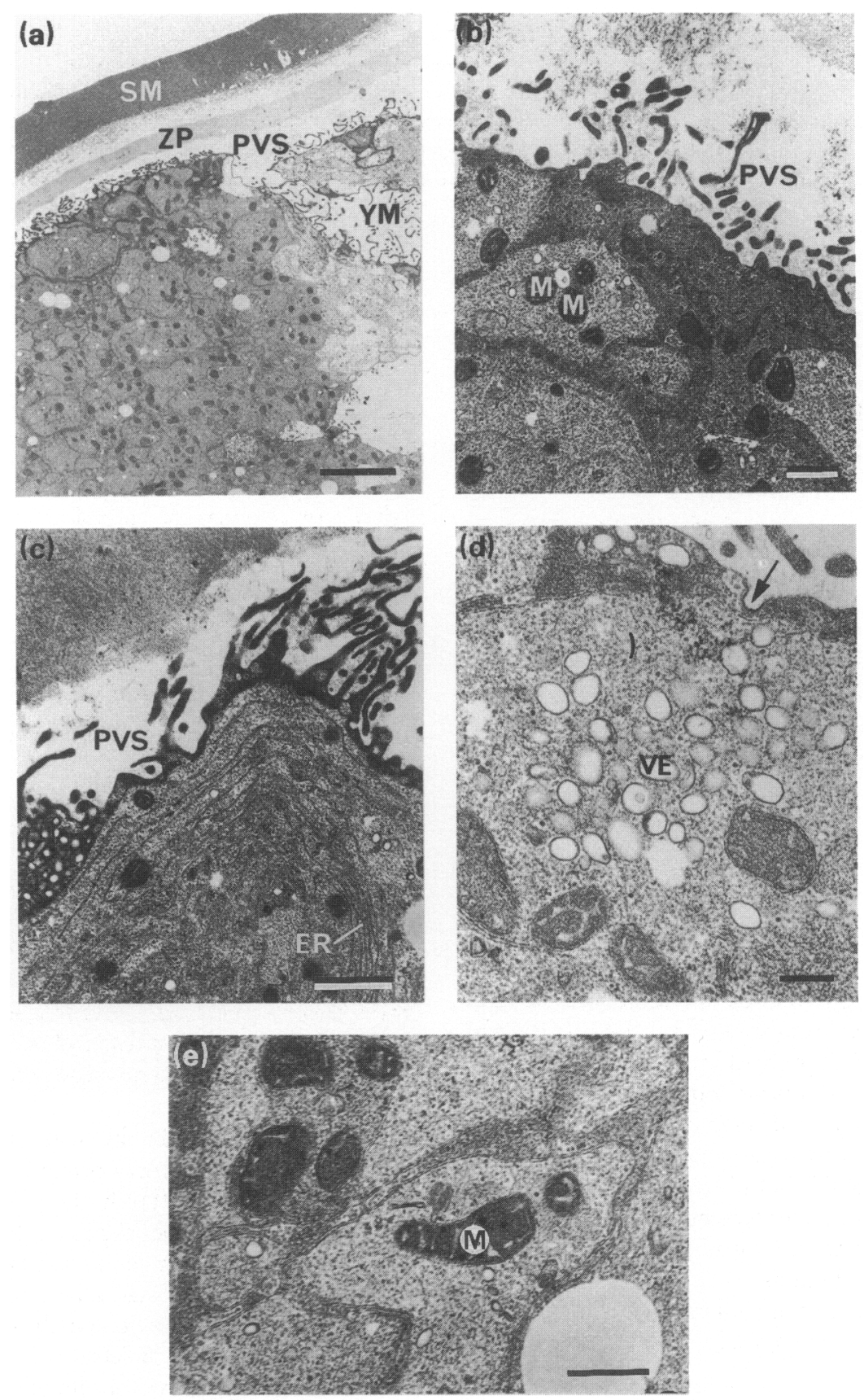

Fig. 1. (a, b, c) Transmission electron microscopy of control and (d, e) DMSO-treated two-cell embryos from Sminthopsis crassicaudata. (a, b) Cytoplasm had abundant mitochondria (M), with the yolk mass (YM) attached to one of the blastomeres. Abundant microvilli protruded into the perivitelline space (PVS) outside which a zona pellucida (ZP), mucoid coat, and shell membrane (SM) occurred. (c) Cytoplasm was of variable electron density; localized groups of vesicles and cisternae of rough endoplasmic reticulum (ER) were sometimes evident. (d, e) Scattered groups of typical vesicles (VE) occur, mitochondria (M) have a dense matrix, and cristae are sometimes a little variable in shape; arrow in (d) indicates endocytotic pit. Scale bars: (a), $5 \mu \mathrm{m}$; (b, c), $1 \mu \mathrm{m}$; (d), $0.25 \mu \mathrm{m}$; (e), $0.5 \mu \mathrm{m}$.

periphery of the blastomeres (Fig. Id). Cisternae of endoplasmic reticulum were often normal, although a few were dilated, and the mitochondria had a typical dense matrix and lamellar cristae (Fig. Ie).
The seven frozen-thawed two-cell embryos varied somewhat in their structural appearance, with blastomeres often appearing slightly shrunken. In some of these embryos, the mitochondria looked typical (Fig. 2a, b, c), although in others 

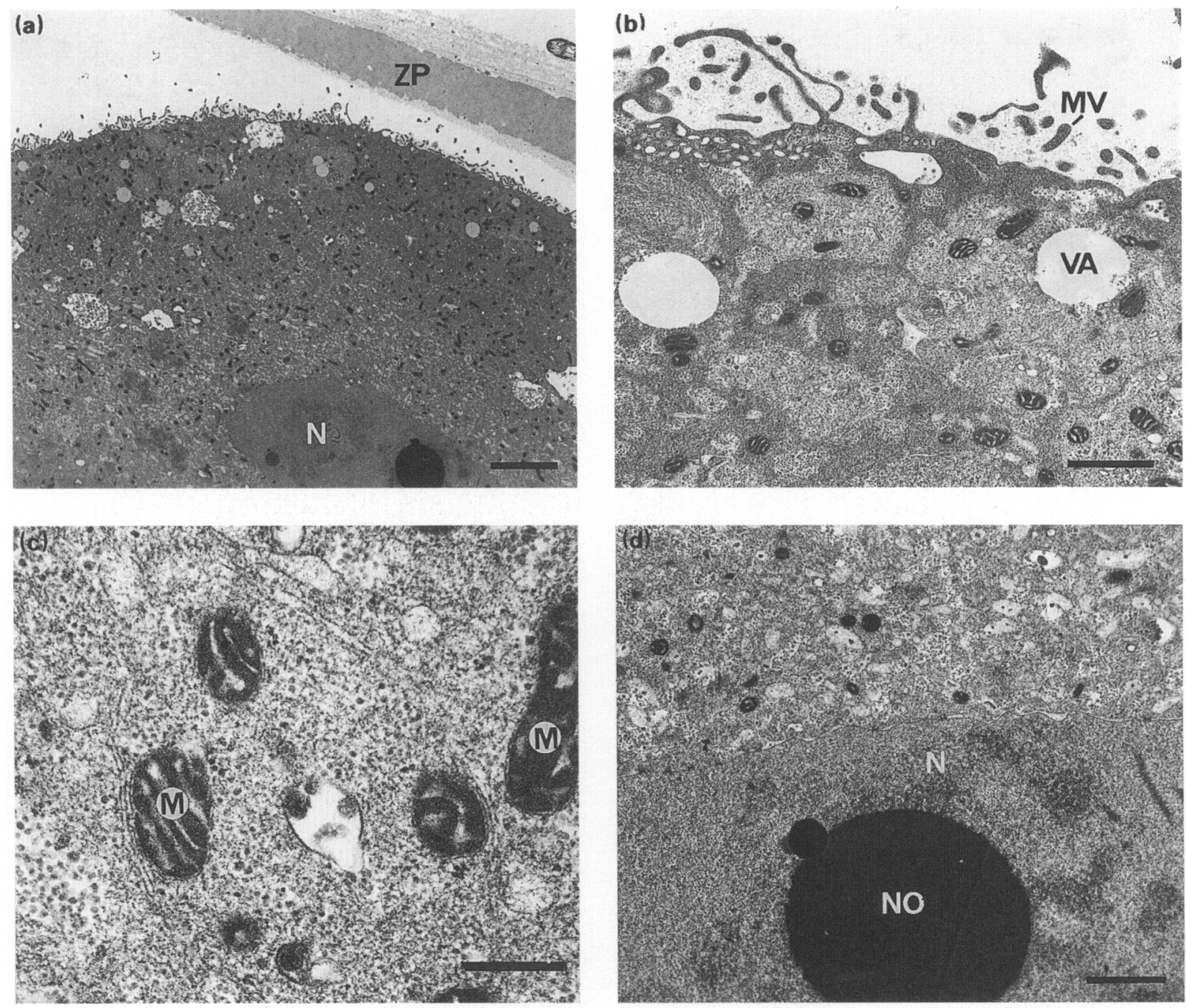

Fig. 2. Transmission electron microscopy of frozen-thawed two-cell embryos from Sminthopsis crassicaudata. (a) A large space sometimes occurs between the cell membrane of the blastomere and the zona pellucida ( $Z \mathrm{P})$. ( $b, c)$ Mitochondria (M) in most cases look typical, although in a few distorted cristae are evident, and cisternae sometimes appear enlarged. MV, microvilli; VA, vacuole. (d) The nucleus $(\mathrm{N})$ has an electron-dense nucleolus $(\mathrm{NO})$, an intact nuclear envelope, and occasional nuclear pores. Scale bars: (a), $5 \mu \mathrm{m}$; (b, c, d), I $\mu \mathrm{m}$.

the cristae appeared enlarged or degenerate. Often the nuclear envelope appeared slightly wrinkled, and the intracytoplasmic membranes sometimes contained what appeared to be dilated segments. However, the nucleolus was normal in appearance and, at least in some, the nuclear envelope was intact and nuclear pores were evident (Fig. 2a, d). Endoplasmic reticulum, fibrous arrays, vacuoles and vesicles, some of which had fused with the cell membrane, were also seen and appeared normal.

Four-cell embryos. In most of the 12 control four-cell embryos, light micrographs indicated that the blastomeres lay close to the inner surface of the zona and, at least in some cases, were joined by a cytoplasmic bridge (Fig. 3a).

Sections through one of the embryos showed that the sperm tail extended into the central cavity between the blastomeres and that the coarse fibres of the principal piece were still largely intact (Fig. 3b).
The ultrastructure of the individual blastomeres appeared similar. The cell membrane had numerous microvilli (Fig. 3c), some of which made contact with the inner surface of the zona. The nucleus, with a slightly wrinkled nuclear envelope, contained a prominent nucleolus and the surrounding cytoplasm contained many vacuoles. The cytoplasm varied in electron density, had a few large vacuoles with a granular appearance (Fig. 3c), typical small mitochondria with dense matrix and lamellar cristae (Fig. 3e), occasional groups of vesicles and scattered cisternae of endoplasmic reticulum. Elaborate fibrous arrays containing a thin, central electron dense band, bordered by a paler region and surrounded by electron dense granular material were also evident (Fig. 3d).

In the nine frozen-thawed four-cell embryos, the blastomeres were usually not in contact with the inner surface of the zona but were still connected by cytoplasmic bridges. There was variability in preservation and cytoplasmic electron density between the embryos. In some, the embryos appeared 

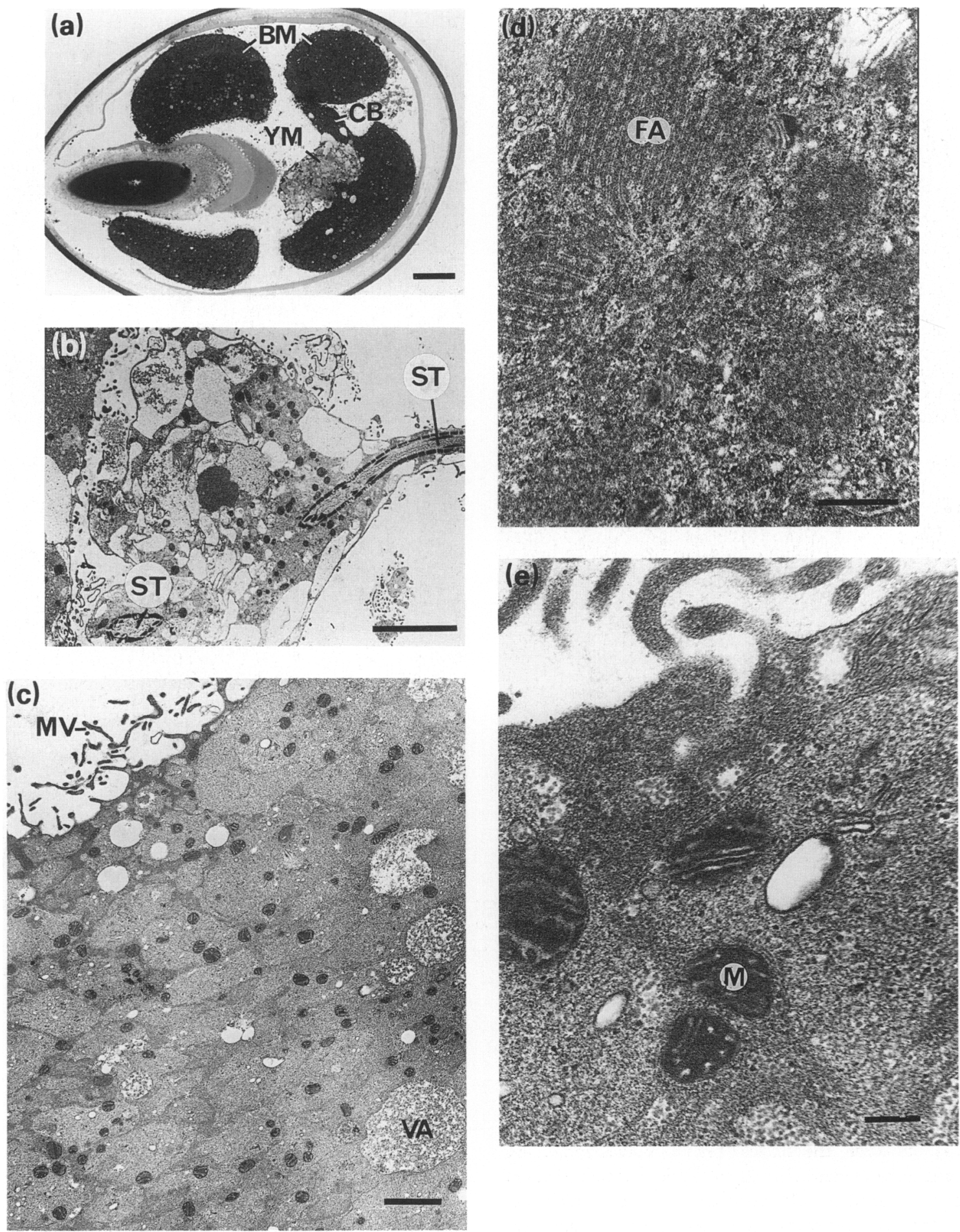

Fig. 3. Four-cell embryos from Sminthopsis crassicaudata (controls). (a) Light microscopy shows that at least two of the blastomeres (BM) are connected by a cytoplasmic bridge (CB) and that the yolk mass (YM) appears to lie inside the blastomeres and is still connected to one of them. The blastomeres have made contact with the inner surface of the zona pellucida. (b-e) Transmission electron micrographs: (b) sperm tail (ST) is still evident and passes into the central cavity of the embryo; (c) one of the blastomeres shows cytoplasm of variable electron density with scattered lipid droplets and vacuoles with flocculent material (VA) (MV, microvilli); (d) scattered fibrous arrays (FA) in cytoplasm of one of the blastomeres; and (e) mitochondria (M) with electron-dense matrix and lamellar cristae. Scale bars: (a), $25 \mu \mathrm{m}$; (b), $5 \mu \mathrm{m}$; (c), $1 \mu \mathrm{m}$; (d), $0.5 \mu \mathrm{m}$; (e), $1 \mu \mathrm{m}$. 

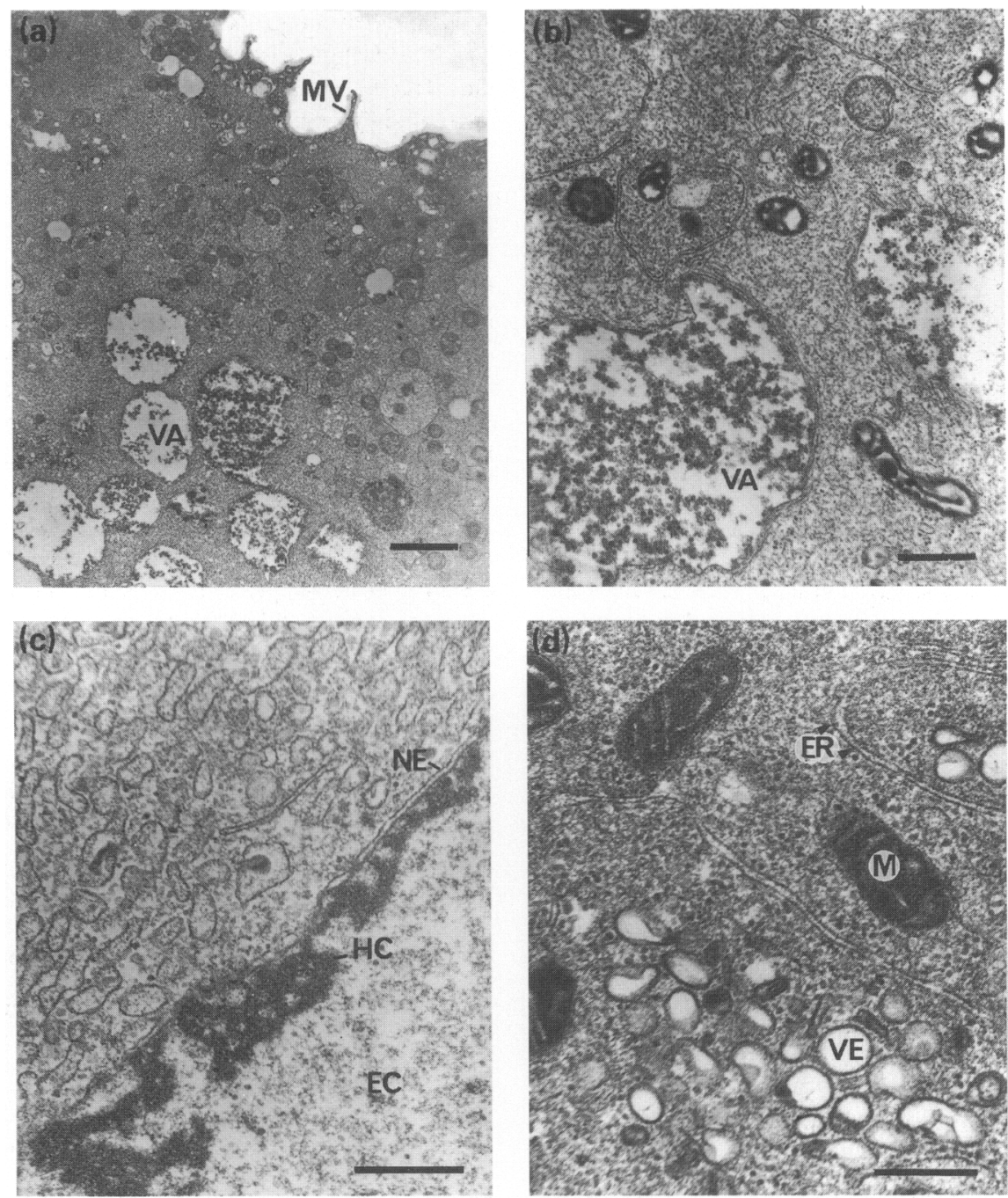

Fig. 4. Transmission electron microscopy of frozen-thawed four-cell embryos from Sminthopsis crassicaudata. (a) Vacuoles (VA) contain granular material and microvilli (MV) are few, short and broad at their base. (b) Large vacuoles (VA) with flocculent material have typical morphology but some mitochondria have distorted lamellar cristae. $(c, d)$ Cisternae of typical endoplasmic reticulum (ER), groups of vesicles (VE), and nucleus with typical nuclear envelope (NE) and heterochromatin ( $\mathrm{HC}$ ) localized to its peripheral region occur. Mitochondria (M) look normal. EC: euchromatin. Scale bars: (a), $0.5 \mu \mathrm{m}$; (b), $0.25 \mu \mathrm{m}$; (c), $0.5 \mu \mathrm{m}$; (d), $1 \mu \mathrm{m}$.

damaged as microvilli tended to be short and fat (Fig. 4a), mitochondria did not appear well preserved or had distorted cristae (Fig. 4b), intracytoplasmic channels and spaces were common, and the nuclear envelopes appeared wrinkled, although they were, nevertheless, intact. In other embryos, very good preservation appeared to have been achieved as the mitochondria contained lamellar cristae with intact membranes, typical large vacuoles occurred, intact vesicles were present, and the matrix was of normal electron density (Fig. $4 \mathrm{~d}$ ). In addition there were cisternae of endoplasmic reticulum that appeared typical, an intact nuclear envelope, and chromatin of normal appearance (Fig. 4c). Elaborate fibrous arrays were observed in sections from approximately half of the embryos examined.

\section{Discussion}

In this study of the dasyurid marsupial Sminthopsis crassicaudata, we investigated whether frozen-thawed embryos could undergo development in vitro and we described the effect of freezing and thawing on blastomere ultrastructure. We showed that some embryos appear morphologically normal after cryopreservation and that a few retained the capacity to undergo cell division.

The cleavage results obtained for non-frozen embryos in culture were slightly lower than those described by Selwood (1987), as we obtained cleavage in only $76 \%$ compared with $>95 \%$ reported by Selwood (1987) using the same methods. This difference is probably partly due to the fact that Selwood 
(1987) induced oestrus and mating by removal of pouch young, whereas we primed females with PMSG. Although using PMSG brings most females into oestrus and ovulation rates are similar to those of females that have had their pouch young removed (Breed and Leigh, 1992), the resultant embryo survival is poor (Rodger et al., 1992).

DMEM proved to be a superior culture medium to HTF, as only $38 \%$ embryos underwent cleavage in HTF compared with $76 \%$ in DMEM. This finding suggests that the more complex DMEM medium contains some factors not present in the HTF that support better embryo survival and development.

The percentage of embryos undergoing cleavage in culture after cryopreservation was markedly lower than that of the non-frozen controls. A comparison of the results obtained using the method of Carroll et al. (1989) indicated that $18 \%$ of frozen-thawed embryos underwent cleavage in culture compared with $38 \%$ of controls incubated in HTF under identical conditions. Although the numbers of embryos are small, they do suggest that freezing and thawing under these conditions is consistent with embryo survival and viability.

Ultrarapid freezing and vitrification methods offer advantages of ease and speed, but embryo development was poor when the method of Kasai et al. (1990) was used compared with the slow freezing method. This finding suggests that cryopreservation using the rapid methods may be harmful to subsequent development; this conclusion is also supported by lack of cleavage of any embryos after using the ultrarapid freezing method of Shaw et al. (1991).

The percentage of embryos developing in culture after cryopreservation was far lower than that previously obtained for mouse embryos (Whittingham et al., 1979; Kasai et al., 1990; Shaw et al., 1991), suggesting that the culture conditions were not optimal for the marsupial embryos. There are a number of differences between these embryos and those of other species that may result in their different sensitivities to cryopreservation. Perhaps the most obvious of these is the considerably greater cell volume of the marsupial embryos compared with those of eutherians. For example, a mouse zygote with a radius of $35 \mu \mathrm{m}$ has a volume of about $1.8 \times 10^{5} \mu^{3}$, compared with a Sminthopsis zygote which with a radius of about $100 \mu \mathrm{m}$, has a volume of approximately $4.2 \times 10^{6} \mu^{3}-$ a difference of about 15 times. In addition, in Sminthopsis PMSG priming produces embryos of variable quality, some of which are clearly grossly abnormal (Rodger et al., 1992). In addition, the large scale empirical studies that have been used to optimize methods in laboratory mice cannot be used in Sminthopsis owing to the small numbers of animals available.

The effect of cryopreservation on embryos frozen-thawed by the method of Carroll et al. (1989) was examined in more detail by fixing some for transmission electron microscopy and comparing their ultrastructure with that of non-frozen controls. The ultrastructure of frozen-thawed embryos was in some cases similar to that of controls, although, in others, abnormalities were found, including dilated channels of endoplasmic reticulum, poorly preserved cristae of the mitochondria, stunted and fewer microvilli, and a wrinkled nuclear envelope with increased heterochromatin around its periphery. It is not clear whether these abnormalities resulted in the apparent low rates of cleavage of embryos after cryopreservation. Exposure to DMSO did not appear generally to result in gross abnormalities, suggesting that DMSO toxicity was not a major factor affecting the normality of frozen-thawed embryos. The ultrastructure of frozen-thawed human embryos has also been examined (Mohr et al., 1985; Ng et al., 1988) and was found to be similar to that of unfrozen controls, although occasional swelling of the endoplasmic reticulum (similar to that in the present study) was observed.

Establishment of routine procedures for the cryopreservation and subsequent development of marsupial embryos could be important for the conservation and management of these species, especially for those with greatly restricted distributions. In this study, we have shown that marsupial embryos can survive cryopreservation and then cleave in culture. However, considerable further refinement of the technique is required before a high rate of success is achieved.

The authors would like to thank J H Bennett of the Department of Genetics for the supply of the animals. This work was funded by an ARC grant (No. A09132094) to the senior author.

\section{References}

Anderson R and Breed WG (1993) In vivo parthenogenetic activation of ovulated oocytes in a dasyurid marsupial, Sminthopsis crassicaudata Zygote $\mathbf{I}$ 231-236

Bennett JH, Breed WG, Hayman DL and Hope RM (1990) Reproductive and genetic studies with a laboratory colony of the dasyurid marsupial Sminthopsis crassicaudata Australian Journal of Zoology 37 207-222

Breed WG and Leigh CM (1990) Morphological changes in the oocyte and its surrounding vestments during in vivo fertilization in the dasyurid marsupial Sminthopsis crassicaudata Journal of Morphology 204 177-196

Breed WG and Leigh CM (1992) Marsupial fertilization: Some further ultrastructural observations of the dasyurid Sminthopsis crassicaudata Molecular Reproduction Development 32 277-292

Carroll J, Warnes GM and Matthews CD (1989) Increase in digyny explains polyploidy after in vitro fertilization of frozen-thawed mouse oocytes Journal of Reproduction and Fertility 85 489-494

Glenister PH, Whittingham DG and Wood MJ (1990) Genome cryopreservation: a valuable contribution to mammalian genetic research Genetic Research 56 $253-258$

Hill JP (1910) The early development of the marsupials, with special reference to the native cat (Dasyurus viverrinus) Quarterly Journal of Microscopical Science 56 I-134

Kasai M, Komi JH, Takakamo A, Tsudera H, Sakurai T and Machida T (1990) A simple method for mouse embryo cryopreservation in a low toxicity vitrification solution, without appreciable loss of viability journal of Reproduction and Fertility $\mathbf{8 9} 91-97$

Mohr LR, Trounson AO and Freeman L (1985) Deep-freezing and transfer of human embryos Journal of In Vitro Fertilization and Embryo Transfer 2 1-10

Ng SC, Sathananthan AH, Wong PC, Ratman SS, Ho J, Mok H and Lee MN (1988) Fine structure of early human embryos frozen with I, 2 propanediol Gamete Research 19 253-263

Quinn P, Barros C and Whittingham DG (1982) Preservation of hamster oocytes to assay the fertilizing capacity of human spermatozoa Joumal of Reproduction and Fertility 66 161-168

Quinn P, Kerin JF and Warnes GM (1985) Improved pregnancy rate in human in vitro fertilization with the use of a medium based on the composition of human tubal fluid Fertility and Sterility 44 493-498

Rodger JC, Breed WG and Bennett JH (1992) Gonadotrophin-induced oestrus and ovulation in the polyovulatory marsupial Sminthopsis crassicaudata Reproduction Fertility and Development 4 145-152

Selwood L (1987) Embryonic development in culture of two dasyurid marsupials, Sminthopsis crassicaudata (Gould) and Sminthopsis macroura (Spencer) during cleavage and blastocyst formation Gamete Research 16 355-370

Selwood L (1989a) Marsupial pre-implantation embryos in vivo and in vitro. In Development of Pre-implantation Embryos and their Environment, pp 225-236 Eds K Yoshinaga and T Mori. Alan Liss, New York 
Selwood L (1989b) Development in vitro of investment-free marsupial embryos during cleavage and early blastocyst formation Gamete Research 23 399-413

Selwood L and Smith D (1990) Time-lapse analysis and normal stages of development of cleavage and blastocyst formation in the marsupials, the Brown Antechinus and the Stripe-faced Dunnart Molecular Reproduction and Development 26 53-62

Selwood L and Young GJ (1983) Cleavage in vivo and in culture in the dasyurid marsupial Antechinus stuartii (Macleay) Journal of Morphology 175 43-60

Shaw JM, Diotallevi L and Trounson AO (1991) A simple rapid 4.5 M Dimethylsulfoxide freezing technique for the cryopreservation of one cell to blastocyst stage preimplantation mouse embryos Reproduction Fertility and Development 3 621-626
Whittingham DG (1971) Survival of mouse embryos after freezing and thawing Nature $233125-126$

Whittingham DG, Leibo SP and Mazur P (1972) Survival of mouse embryos frozen to $-196^{\circ} \mathrm{C}$ and $-269^{\circ} \mathrm{C}$ Science $187411-414$

Whittingham DG, Wood M, Farrant J, Lee H and Halsey JA (1979) Survival of frozen mouse embryos after rapid thawing from $-196^{\circ} \mathrm{C}$ Journal of Reproduction and Fertility 56 11-21

Wilmut I (1972) The effect of cooling rate, warming rate, cryoprotective agent and stage of development on survival of mouse embryos during freezing and thawing Life Sciences 11 1071-1079 